Revue internationale P.M.E.

Économie et gestion de la petite et moyenne entreprise

\title{
Profils de dirigeants et données comptables de gestion en PME
}

\section{Philippe Chapellier}

Volume 10, numéro 1, 1997

URI : https://id.erudit.org/iderudit/1009016ar

DOI : https://doi.org/10.7202/1009016ar

Aller au sommaire du numéro

\section{Éditeur(s)}

Presses de l’Université du Québec

ISSN

0776-5436 (imprimé)

1918-9699 (numérique)

Découvrir la revue

Citer cet article

Chapellier, P. (1997). Profils de dirigeants et données comptables de gestion en PME. Revue internationale P.M.E., 10(1), 9-41. https://doi.org/10.7202/1009016ar

\section{Résumé de l'article}

Selon la littérature se rapportant au système de gestion du dirigeant de PME, bon nombre d'entreprises de taille petite ou moyenne ne disposeraient que d'outils de gestion embryonnaires, ce qui expliquerait l'intérêt des dirigeants de PME pour les médias plus informels, pour les informations verbales. Toutefois, les études empiriques réalisées sur ce thème mènent à des conclusions nuancées. Ainsi, la vision d'un système de données comptables de gestion (SDCG) rudimentaire ne correspond pas à la réalité de toutes les PME observées, les dirigeants de PME ayant, face à ce type de données, des attitudes différenciées. À côté de cela, de nombreux spécialistes expliquent qu'un élément tout à fait caractéristique de l'univers des PME réside dans l'extrême diversité des profils de dirigeants. Dans ce contexte, l'objectif de cet article consiste à essayer de déterminer dans quelle mesure le profil des dirigeants de $\mathrm{PME}$ « guide » leur comportement face aux données comptables de gestion. Pour répondre à cet objectif, une enquête a été réalisée par entretien direct et par administration d'un questionnaire auprès d'un échantillon de 113 dirigeants de PME. L'étude révèle que le profil d'un dirigeant est significativement lié à son type de pratiques comptables : les dirigeants de profils différents disposent de SDCG de complexité différente et utilisent les données comptables de manière différenciée.
Ce document est protégé par la loi sur le droit d'auteur. L’utilisation des services d’Érudit (y compris la reproduction) est assujettie à sa politique d'utilisation que vous pouvez consulter en ligne.

https://apropos.erudit.org/fr/usagers/politique-dutilisation/ 


\title{
Profils de dirigeants et données comptables de gestion en PME
}

Philippe CHAPELLIER

Université de Montpellier II

MOTS CLÉS

\section{Profils de dirigeants - Données comptables de gestion Systèmes d'information - PME}

\begin{abstract}
RÉSUMÉ
Selon la littérature se rapportant au système de gestion du dirigeant de PME, bon nombre d'entreprises de taille petite ou moyenne ne disposeraient que d'outils de gestion embryonnaires, ce qui expliquerait l'intérêt des dirigeants de PME pour les médias plus informels, pour les informations verbales. Toutefois, les études empiriques réalisées sur ce thème mènent à des conclusions nuancées. Ainsi, la vision d'un système de données comptables de gestion (SDCG) rudimentaire ne correspond pas à la réalité de toutes les PME observées, les dirigeants de PME ayant, face à ce type de données, des attitudes différenciées. À côté de cela, de nombreux spécialistes expliquent qu'un élément tout à fait caractéristique de l'univers des PME réside dans l'extrême diversité des profils de dirigeants. Dans ce contexte, l'objectif de cet article consiste à essayer de déterminer dans quelle mesure le profil des dirigeants de PME " guide " leur comportement face aux données comptables de gestion. Pour répondre à cet
\end{abstract}

\section{L'AUTEUR}

Philippe Chapellier est maître de conférences à l'Université de Montpellier II. II est membre du CREGO (Centre de recherche en gestion des organisations) à l'IAE de Montpellier et membre du GREFICCO (Groupe de recherche en finance, comptabilité et contrôle). Ses activités de recherche actuelles portent sur le système d'information du dirigeant de PME. Adresse: IUT de Montpellier, Département d'informatique, 99, avenue d'Occitanie, 34096 Montpellier, cedex, France. 
objectif, une enquête a été réalisée par entretien direct et par administration d'un questionnaire auprès d'un échantillon de 113 dirigeants de PME. L'étude révèle que le profil d'un dirigeant est significativement lié à son type de pratiques comptables: les dirigeants de profils différents disposent de SDCG de complexité différente et utilisent les données comptables de manière différenciée.

\begin{abstract}
Literature on the management system of small and medium businesses (SMB) managers shows that many SMB have only embryonic management tools and mentions SMB managers' attraction for informal media and verbal information. However, empirical studies have come to somewhat different conclusions: the vision of a rudimentary management accounting data system (MADS) does not correspond with the real nature of all SMB, the managers of SMB are reported to have differentiated attitudes towards such data. Moreover, many specialists explain that the specificity of SMB lies in the wide range of managers' profiles. In this context, the paper aims at determining to what extent SMB managers' profiles can account for their attitudes as regards management accounting data. To achieve this objective, a survey has been conducted by means of direct interviews with 113 managers of small and medium independent businesses. The results show a close relationship between managers' profiles and types of management accounting practices: managers with diverse profiles have MADS of different complexity and use management accounting data differently.
\end{abstract}

\title{
RESUMEN
}

La literatura que trata el tema del sistema de gestión del dirigente de PyME deja aparecer que numerosas PyMEs sólo disponen de instrumentos de gestión embrionarios y apunta la atracción de los dirigentes de PyME por medios de información mucho más informales, por las informaciones verbales. Sin embargo, los estudios empíricos realizados en el tema revelan conclusiones matizadas, la vision de un sistema de dadas contables de gestión (SDCG) rudimentario no corresponde a la realidad de todas las PyMEs observadas, los dirigentes de PyME tendrian, frente a ese tipo de dadas, actitudes diferenciadas. Por otra parte, numerosos especialistas explican que uno de los elementos más característicos del mundo de las PyMEs radica en la diversidad de los perfiles de dirigente que uno puede observar. En este contexto, el objectivo de este artículo consiste en intentar determinar en que medida el perfil de los dirigentes de PyME "guía " su comportamiento frente a las dadas contables de gestion. Para responder a este objectivo, una encuesta ha sido realizada por medio de un cuestionario al cual han respondido 113 dirigentes de PyME durante entrevistas directas. El estudio revela que el perfil de los dirigentes es significativamente relacionado con su tipo de prácticas contables: los dirigentes con perfiles diferentes disponen de SDCG de complejidad diferente y utilizan las dadas contables de manera diferenciada. 


\section{Problématique}

Selon la littérature se rapportant au système de gestion du dirigeant de PME, bon nombre d'entreprises de taille petite ou moyenne ne disposeraient que d'outils de gestion embryonnaires, ce qui expliquerait l'intérêt des dirigeants de PME pour les médias plus informels, pour les informations verbales (Mintzberg, 1990 ; Fallery, 1983a). De façon générale, les dirigeants de PME auraient, pour prendre leurs décisions, exclusivement recours à leurs seuls jugements, intuitions et expériences (Mintzberg, 1976 ; Simon, 1987 ; Nadeau et al., 1987) et auraient, pour la plupart, une propension à penser qu'ils sont capables de gérer leur affaire seuls, sans autre système d'information de gestion que celui constitué par quelques données comptables qu'ils jugent essentielles (Dupuy, 1987). Toutefois, les études empiriques réalisées sur le thème des pratiques comptables de gestion en PME révèlent des conclusions nuancées (Robinson et Pearce, 1984 ; de Thomas et Fredenberger, 1985 ; Orpen, 1985 ; Robinson et al., 1986a, 1986b ; Riggs et Bracker, 1986 ; Gable et Topol, 1987 ; Holmes et Nicholls, 1988 ; Gasse, 1989 ; d'Amboise et Bakanibona, 1990 ; Rasolofoson, 1993 ; Fernandez et al., 1994). La vision d'un système de données comptables de gestion (SDCG) rudimentaire ne correspond pas à la réalité de toutes les PME observées et si effectivement certains dirigeants ne vont pas au-delà de la simple production des données comptables obligatoires, d'autres en revanche disposent de systèmes plus complexes. Les dirigeants de PME auraient, face à ce type de données, des attitudes différenciées.

À côté de cela, de nombreux spécialistes de la PME expliquent qu'un élément tout à fait caractéristique de l'univers des PME réside dans l'extrême diversité des profils de dirigeants, «parfois autodidacte, parfois diplômé de l'enseignement supérieur, tour à tour jeune créateur sans expérience, cadre démissionnaire d'un grand groupe ou bien héritier d'une vieille affaire familiale », Naro (1989) explique qu'il est bien difficile d'établir une image type du dirigeant de PME et que cette hétérogénéité dans le profil des dirigeants rend plus difficile encore la compréhension des logiques de comportement qui guident les PME ; « quoi de commun en effet entre le mineur licencié qui ouvre une épicerie de village, l'étudiant au chômage qui offre un service de photocopies près de son école et le jeune ingénieur qui monte une entreprise d'électronique très spécialisée dans une pépinière d'entreprises ? » s'interrogent Julien et Marchesnay (1990). Dans ce contexte, l'objectif essentiel de la recherche consiste à essayer de déterminer dans quelle mesure le profil des dirigeants de PME « guide » leur comportement face aux données comptables (entendues en termes de données de gestion interne : données sur les coûts, budgets, analyse de rentabilité, analyse du risque de défaillance, tableaux de bord, situations intermédiaires). 
Avant d'aller plus loin dans la détermination de la problématique, il convient de préciser la définition de la PME et des principaux concepts de la recherche avec rigueur et ce faisant, d'établir les limites de notre champ d'investigation.

La difficulté de définir la PME tient à la grande variété des caractéristiques de ce type d'entreprises. Diverses études ont mis en évidence l'influence personnelle du dirigeant-propriétaire, son autonomie décisionnelle, la propriété des capitaux, la forte influence de l'environnement, la faiblesse des parts de marché, l'aspect spécialisé des activités, etc. (Julien et Marchesnay, 1988). Notre objectif n'est pas de reprendre ici l'ensemble de la littérature, assez abondante, qui existe sur le thème des critères d'identification des PME, mais plus simplement de retenir une définition synthétique qui permettra de délimiter le champ de notre étude. Ainsi, nous entendrons par PME «toute entreprise juridiquement indépendante disposant d'un effectif compris entre 10 et 100 salariés ». D'une manière très classique, les critères retenus concernent, d'une part, le nombre de salariés et, d'autre part, l'indépendance de l'entreprise en termes de capital. Sont donc exclues de notre champ de recherche les très petites entreprises (TPE), ayant moins de 10 salariés, les entreprises de plus de 100 salariés, ainsi que les filiales, succursales ou divisions d'entreprises plus importantes.

La définition des «pratiques comptables de gestion» retenue a pour cadre de référence la théorie subjectiviste. Le constat de départ de cette définition est que le langage du management, celui de l'informatique ainsi que le langage courant utilisent sans grande précaution, sans grande précision, les termes de donnée et d'information. De même, rares sont les recherches empiriques distinguant clairement la production des données comptables de leur utilisation. Souvent, en effet, les concepts d'information et de donnée sont considérés comme équivalents et font l'objet de confusion que des auteurs comme Mélèse (1979), Bateson, (1977) ou Simon (1974) se sont pourtant attachés à dissiper. Cette distinction nous semble toutefois essentielle (Reix, 1987 ; Dupuy, 1994 ; Chapellier et de Montgolfier, 1995) et explique notre décision d'envisager les pratiques comptables simultanément en termes de données disponibles et de données utilisées. Ainsi, les pratiques comptables de gestion sont définies par deux dimensions :

- une dimension "objective » : un système de données disponibles, à base comptable, historiques ou prévisionnelles, qui recouvre à la fois la comptabilité générale, le contrôle de gestion, l'analyse financière et l'élaboration de tableaux de bord comptables;

- une dimension "subjective " : l'utilisation de ces données par le dirigeant de PME en réponse à ses besoins. Cette dimension nous permettra 
de juger de la pertinence des données comptables en PME. L'approche retenue de la problématique de la pertinence est donc celle de l'utilisation : si le dirigeant utilise les données, c'est qu'elles sont pertinentes.

Dans ce contexte, les données comptables renvoient aux données de gestion interne qui, élaborées en totalité ou en partie sur la base de la comptabilité, sont disponibles dans l'entreprise, et les informations comptables représentent les données comptables qui contribuent aux processus de prise de décision des dirigeants (Dupuy, 1994), c'est-à-dire les données comptables effectivement utilisées. Dans cette perspective, la donnée est donc comprise comme la matière brute nécessaire à la production d'informations. Elle se mute en information lorsqu'elle apporte un message, lorsqu'elle a une valeur sémantique pour son destinataire (Davis et al., 1986).

Une fois le champ d'investigation et les différents concepts clairement définis, les objectifs de la recherche peuvent être exposés de manière plus affinée. Ainsi, le premier objectif de la recherche consiste à élaborer une typologie des dirigeants de PME qui, malgré son caractère réducteur, devrait permettre de faire face à la complexité liée à l'hétérogénéité et de mieux saisir la variété des profils observés. L'une des typologies à laquelle il est le plus souvent fait référence est celle élaborée par Smith (1967) qui opère une distinction entre l'entrepreneur «artisan» présentant une formation technique, motivé par un ardent désir d'indépendance, mais aussi de pérennité et de stabilité, et l'entrepreneur « opportuniste», stimulé par un assez vaste éventail culturel, socialement éveillé, flexible, confiant, orienté vers le futur, caractérisé par une formation de type gestionnaire et plus apte à recourir aux pratiques modernes de gestion. Au-delà de cette opposition, la réalité semble toutefois beaucoup plus nuancée. Il est d'ailleurs à noter que Smith (dans Smith et al., 1992) a récemment proposé d'enrichir sa typologie en y incorporant un nouveau type, soit le dirigeant «innovateur » (inventor entrepreneur). Bien entendu, de nombreux auteurs ont proposé d'autres typologies (Laufer, 1974 ; Macintosh, 1987 ; Julien et Marchesnay, 1988); compte tenu de la littérature existant sur le thème, nous tenterons d'élaborer une typologie des chefs de PME, c'est-àdire de relever, parmi les dirigeants de notre échantillon, différents groupes d'individus ayant des caractéristiques en commun qui les distinguent des autres groupes.

Le second objectif de la recherche consiste à repérer et à décrire les pratiques comptables des dirigeants de PME. Il s'agira de caractériser les données comptables disponibles en PME et de relever dans quelle mesure et dans quelles circonstances les dirigeants de PME consomment, dans leur travail de chef d'entreprise, de l'information comptable. Ensuite, une étude des relations entre le degré de complexité du SDCG et le degré d'utilisation des données comptables par le dirigeant sera réalisée afin de déterminer dans quelle 
mesure les données disponibles sont effectivement utilisées. Nous tâcherons enfin de repérer des similitudes dans l'ensemble des cas observés pour proposer une typologie des pratiques comptables des dirigeants de PME, c'est-à-dire d'essayer d'identifier différents types de pratiques comptables propres à la PME.

Le troisième objectif de la recherche consiste à étudier la relation entre les profils de dirigeants et leurs pratiques comptables de gestion. Les pratiques comptables de gestion des dirigeants de PME sont-elles différenciées ? Si nous répondons par l'affirmative, cette question se dédouble alors en pourquoi et comment? Épistémologiquement, deux perspectives aux implications théoriques distinctes peuvent être relevées dans la littérature. La première est la théorie de la contingence structurelle qui peut être qualifiée d'objectiviste. Cette théorie pose, en effet, l'existence d'un lien entièrement déterminé entre la structure des organisations et les traits qui caractérisent les situations dans lesquelles elles opèrent, et nie de fait l'existence potentielle d'influences de la part des acteurs. Or, l'influence du dirigeant de PME dans la gestion de son entreprise constitue précisément l'une des caractéristiques essentielles de ce type d'entreprises (Fallery, 1983b ; Specht, 1987 ; Marchesnay, 1988 ; Julien et Marchesnay, 1988 ; Lorrain et Dussault, 1988 ; d'Amboise, 1989 ; Lefebvre 1991 ; Woo et al., 1991; Marchesnay, 1992a). C'est pourquoi il convient à notre sens d'élargir le cadre de recherche à la théorie subjectiviste selon laquelle les caractéristiques comportementales propres à chacun des acteurs sont susceptibles d'influencer la structure et le fonctionnement des entreprises. Cette approche admet le principe qu'un individu est actif par rapport à la situation et non contraint par elle ou, plus précisément, que si les caractéristiques organisationnelles et de contexte peuvent, en effet, dans une certaine mesure, limiter et déterminer ses choix, l'acteur n'en est pas moins libre et doué d'intentionnalité et dispose d'une marge de liberté (Crozier et Friedberg, 1977). Notre thèse réalisée sur le thème révèle que si les caractéristiques organisationnelles (et tout particulièrement la taille des entreprises) sont, jusqu'à un certain point, liées aux pratiques comptables de gestion des dirigeants de PME, celles-ci ne peuvent seules, expliquer l'ensemble de ces pratiques (Chapellier, 1994). C'est pourquoi nous avons choisi dans le cadre de cet article de nous concentrer exclusivement sur des variables de type comportemental propres à l'acteur essentiel en PME, à savoir le dirigeant. L'objectif consiste ici à essayer de déterminer dans quelle mesure le profil des dirigeants de PME est associé à leurs pratiques comptables de gestiọn.

Au total, l'objectif de la recherche consiste donc à élaborer une typologie de dirigeants de PME, une typologie de leurs pratiques comptables de gestion afin d'étudier ensuite la relation existant entre les profils de dirigeants repérés et les pratiques comptables. Cela nous conduit dès lors à présenter la méthodologie employée pour tenter d'atteindre ce triple objectif. 
FIGURE 1

Le cadre de recherche

OBJECTIF N $^{\circ} 1$ : ÉTUDE DES PRATIQUES COMPTABLES DE GESTION DES DIRIGEANTS DE PME

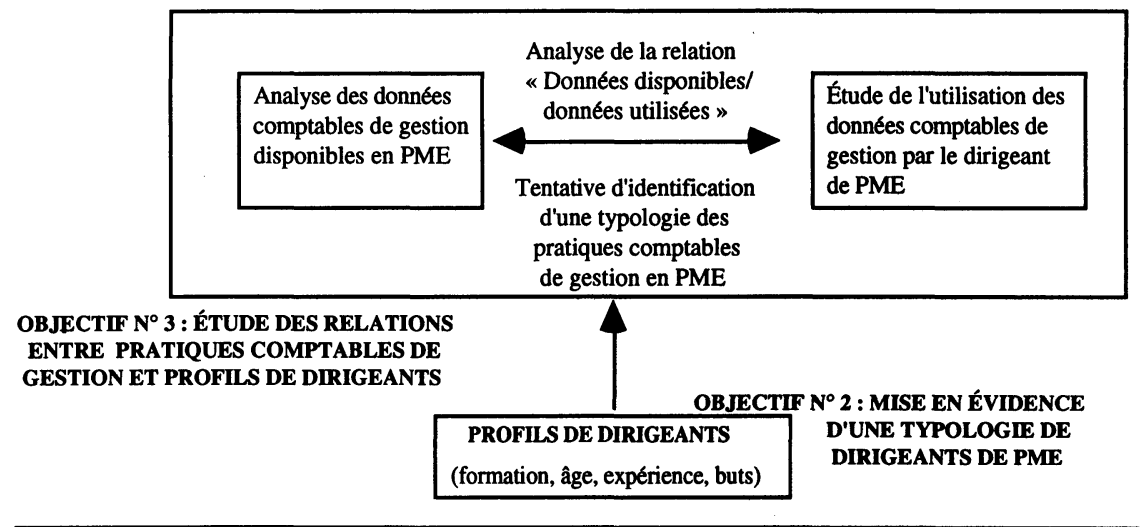

\section{Méthodologie}

Une enquête a été réalisée dans le cadre d'une thèse de doctorat (Chapellier, 1994) par administration, par entretien direct, d'un questionnaire auprès d'un échantillon de 113 dirigeants de PME juridiquement indépendantes et disposant d'un effectif compris entre 10 et 100 salariés. Ce type de méthode, à savoir la réalisation d'études transversales, semble particulièrement adapté quand l'objectif de la recherche consiste, comme c'était le cas ici, à repérer des régularités dans les pratiques. En outre, elle a permis d'accumuler, à travers des commentaires formulés par les dirigeants, diverses informations, divers sentiments qui permettent au chercheur de mieux comprendre le fonctionnement de l'entreprise.

Elle n'est toutefois pas sans difficulté, ni sans limite. Tout d'abord, les données recueillies par le questionnaire sont autorapportées et souvent de l'ordre de la perception. Bien que ce genre d'étude soit très légitime et courant, bien que toutes les enquêtes aient été menées par la même personne, ce qui limite les biais perceptifs, et bien que les comptables salariés aient été interrogés à des fins de validation, des risques de décalage entre «la réalité » et «la perception de cette réalité » persistent, ce qui doit bien entendu nous inciter à la prudence dans l'interprétation des résultats. Par ailleurs, cette méthode se heurte au problème de l'opérationnalisation des différents concepts, et notamment, des pratiques comptables en PME. Il s'agissait en effet de transformer les variables retenues sous forme d'indicateurs et de s'assurer que chacune des mesures utilisées était fidèle, c'est-à-dire mesurait avec précision et de façon constante le construit étudié. 
Nous avons vu que les pratiques comptables de gestion sont définies simultanément en termes de données disponibles et de données utilisées. Ainsi, le SDCG a été caractérisé par son degré de complexité (Kandhwalla, 1972; Rosenzweig, 1977 ; Elmore, 1986 ; Abdou, 1991 ; Chapellier et de Montgolfier, 1995) et, plus précisément, au regard de la diversité, du degré de détail, de la fréquence et du délai de production, des données de comptabilité générale, de contrôle de gestion, d'analyse financière et du tableau de bord (figure 2). La partie du questionnaire permettant de caractériser la complexité du SDCG se trouve en annexe $\mathrm{A}$.

FIGURE 2

Caractérisation

du degré de complexité du SDCG

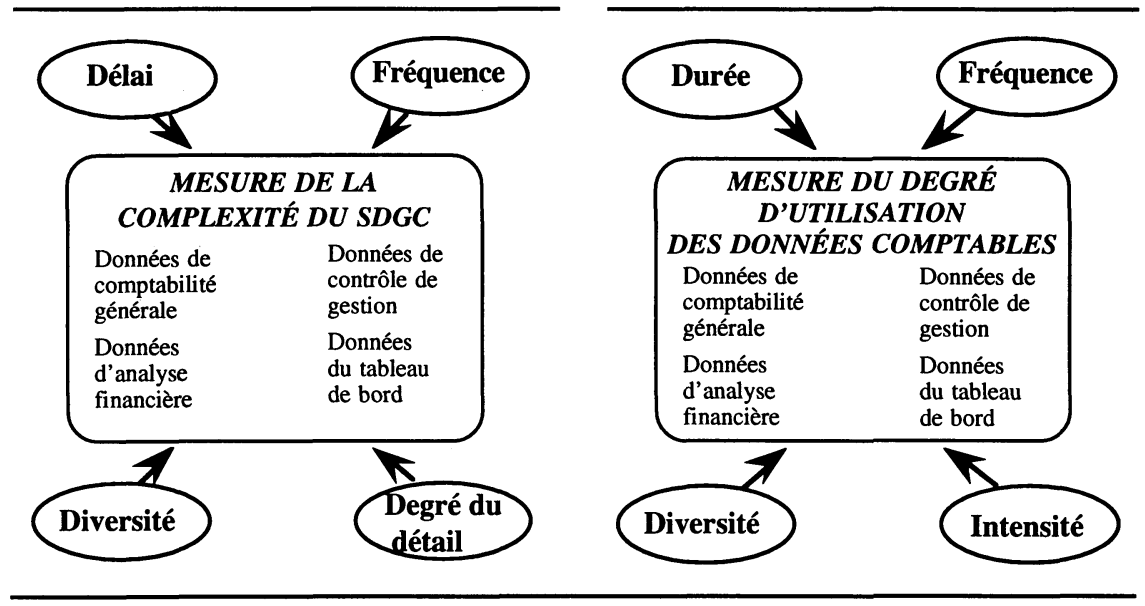

FIGURE 3

Caractérisation du degré d'utilisation des données comptables par le dirigeant de PME
La mesure de l'utilisation a, quant à elle, été abordée sous l'angle du degré d'utilisation apprécié par la fréquence, la durée moyenne hebdomadaire, la diversité et l'intensité de l'utilisation de ces mêmes données (figure 3). La partie du questionnaire permettant de caractériser le degré d'utilisation des données comptables de gestion par le dirigeant de PME se trouve en annexe B.

La fidélité de l'instrument de mesure a été testée et les résultats obtenus permettent de considérer cet outil, élaboré à la base de manière assez intuitive, comme relativement bien adapté. Il possède une bonne cohérence interne, il nous est de ce fait possible de prendre la somme des scores des énoncés comme mesure synthétique de la complexité globale des SDCG, d'une part, et du degré d'utilisation des données comptables de gestion par le dirigeant de PME, d'autre part. 
Enfin, un examen de la littérature nous a permis de définir les variables caractéristiques des chefs de PME à retenir dans le cadre de recherche. D'une manière très classique, les caractéristiques descriptives du profil du dirigeant retenues sont relatives, d'une part, à sa formation (Martel et al., 1985; Hess, 1987 ; Raymond, 1988a, 1988b ; Nadeau et al., 1988 ; Mintzberg, 1988 ; Julien et Marchesnay, 1988 ; Carland et al., 1989 ; Holmes et Nicholls, 1989 ; Holmes et al., 1991), à son âge (Lucas, 1975 ; Reix, 1984 ; Nadeau et al., 1988) et à son expérience (Reix, 1981, 1984 ; Martel et al., 1985 ; Nelson, 1987 ; Nadeau et al., 1988), soit trois caractéristiques relativement objectives et, d'autre part, à ses buts (Marchesnay, 1982, 1990, 1993 ; Mintzberg, 1986 ; Hamilton, 1987 ; Solomon et Fernald, 1988 ; Slatter et al, 1988 ; Blais et Toulouse, 1990 ; Tagiuri et Davis, 1992), soit une caractéristique plutôt subjective. Notons que la vision du dirigeant adoptée reste dans l'ensemble assez « externalisée »; les aspects psychologiques de sa personnalité, tels que son attitude face au risque, sa créativité, sa culture, etc., ne sont notamment pas intégrés au modèle. Les mesures relatives à chacune des variables «objectives » (formation, âge, expérience) restent très classiques (annexe $\mathrm{C}$ ). Les mesures relatives aux buts susceptibles d'être poursuivis par les dirigeants méritent, quant à elles, quelques approfondissements. La littérature sur ce thème met en avant quatre types de buts principaux : la recherche d'indépendance, la volonté de pérennité et de stabilité, la volonté de croissance et la recherche de profit. Il s'agissait de prendre en considération chacune de ces dimensions. Mais il est évident que ces buts peuvent être intimement liés et de plusieurs façons. La croissance peut être nécessaire à la survie, tout comme la survie est nécessaire à la croissance. De même, la poursuite obsessionnelle de l'un des buts peut entrer en conflit avec la poursuite des autres. Par exemple, une croissance trop forte peut freiner le profit et peut-être même la survie et une obsession de survie peut, en fait, faire échouer l'entreprise et aller à l'encontre... de la survie elle-même (Mintzberg, 1986). En réalité, il existerait un «panier de buts » à l'intérieur duquel s'exercerait une certaine hiérarchie et cette hiérarchie s'imprimerait dans la logique des comportements (Naro, 1989). C'est cette hiérarchie que nous avons cherché à mettre en relief. Pour cela, les buts des dirigeants ont été mesurés à l'aide d'échelles à somme constante ou échelles cardinales fortes (on obtient donc des données assimilables à des données quantitatives). Deux séries de quatre énoncés ont été élaborées. Il s'agissait pour les dirigeants de répartir 100 points entre chacun des quatre énoncés correspondant respectivement aux objectifs d'indépendance, de stabilité et de pérennité, de croissance et de profit, en affectant le plus grand nombre de points aux buts qui leur paraissaient les plus importants et de recommencer la même opération avec la seconde série de quatre énoncés (annexe C). La fidélité de la mesure a été testée en calculant la corrélation, pour chacun des quatre types de buts, entre les deux énoncés. Les coefficients obtenus sont tous significatifs à $99,9 \%$ excepté celui mettant 
en relation les deux énoncés mesurant le désir d'indépendance qui n'est significatif qu'à $95 \%$. La fidélité de l'instrument est donc acceptable et nous permet de construire une échelle de score.

Une fois la problématique et la méthodologie exposées, il nous est possible de présenter les principaux résultats de la recherche.

\section{Résultats}

Les résultats de l'analyse descriptive, d'une part, et contingente, d'autre part, des dirigeants en PME et des pratiques comptables de gestion sont successivement présentés.

\subsection{La mise en évidence de trois profils de dirigeants}

L'objectif est d'élaborer, à partir des données recueillies, une typologie de dirigeants de PME, c'est-à-dire d'isoler, parmi l'ensemble des 113 dirigeants de l'échantillon, différents groupes dont les caractéristiques de formation, d'âge, d'expérience et de buts sont relativement identiques entre eux et différentes de celles des autres groupes.

Avant toute chose, rappelons dans un tableau l'ensemble des variables retenues ainsi que leurs modalités.

TABLEAU 1

Récapitulatif des variables retenues

\begin{tabular}{lccc}
\hline & Classe 1 & Classe 2 & Classe 3 \\
\hline Âge du dirigeant & De 20 à 39 ans & De 40 à 49 ans & 50 ans et plus \\
Expérience & De 0 à 9 ans & De 10 à 19 ans & 20 ans et plus \\
Niveau de formation & Autodidacte ou secondaire & Bac + 2 ou 3 & Bac + 4 ou supérieur \\
Type de formation & Gestionnaire & Non gestionnaire & \\
Suivi de stages de gestion & Oui & Non & \\
Objectif d'indépendance & Négligeable & Moyen & Important \\
Objectif de stabilité/pérennité & Négligeable & Moyen & Important \\
Objectif de croissance & Négligeable & Moyen & Important \\
Objectif de profit & Négligeable & Moyen & Important \\
\hline
\end{tabular}

Une analyse factorielle des correspondances multiples (AFCM) nous a permis, dans un premier temps, d'identifier différents groupes homogènes de modalités. Elle nous donne une vision globale et synthétique de la structure des données relatives aux caractéristiques des dirigeants et débouche sur la réalisation d'une analyse typologique permettant quant à elle l'identification 
de différents profils de dirigeants. Le nombre d'axes retenus pour la conception de la classification hiérarchique (opérée sur le logiciel d'analyse de données Chadoc) est de trois, et ces trois axes expliquent à eux seuls 53,64\% de l'inertie totale. La coupure de l'arbre selon la règle des deux nœuds consécutifs entre lesquels la variation de l'indice de niveau est la plus forte (Fenneteau et Biales, 1993) fait apparaître trois profils de dirigeants (coupure à 0,5 ).

FIGURE 4

Représentation graphique des profils de dirigeants identifiés

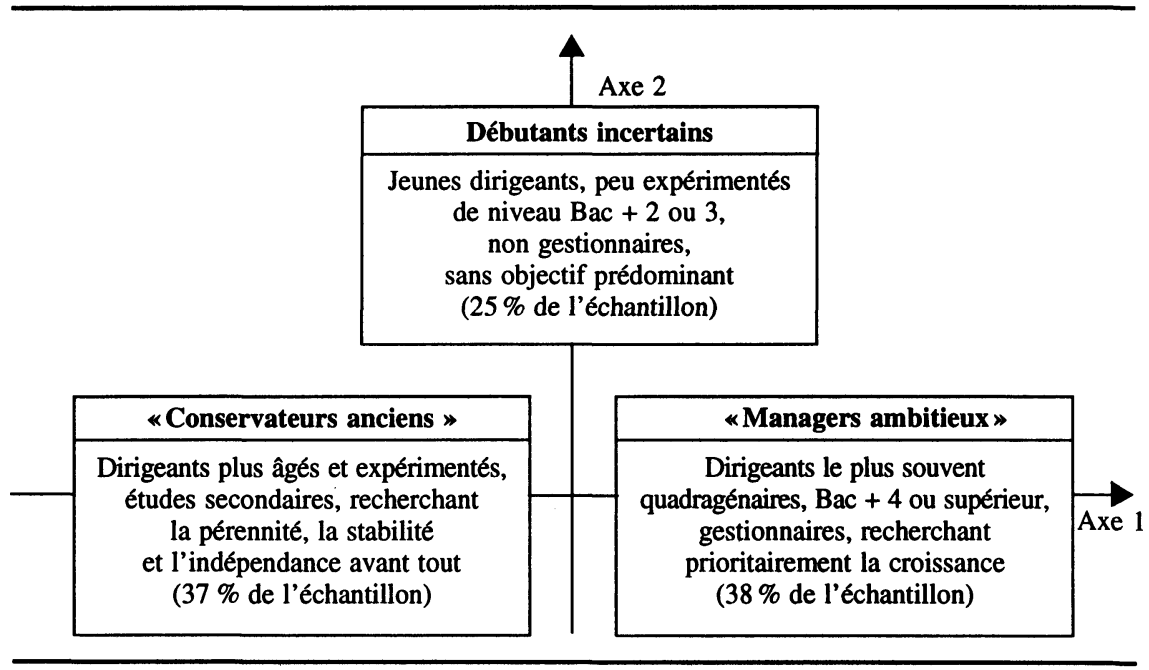

Type 1 : Les «managers ambitieux» : il s'agit de dirigeants le plus souvent quadragénaires, moyennement expérimentés, disposant systématiquement d'une formation de niveau élevé, souvent de type gestionnaire, qui ont participé pour plus de $55 \%$ à des stages de gestion au cours des cinq dernières années et semblent rechercher en priorité la croissance. Ce type de dirigeants représente $38 \%$ de l'échantillon.

Type $2:$ Les « débutants incertains » : il s'agit de jeunes dirigeants, peu expérimentés, disposant le plus souvent d'une formation de niveau $\mathrm{Bac}+2$ ou 3, de type autre que gestionnaire, et seuls $35 \%$ d'entre eux ont participé à un stage de gestion au cours des cinq dernières années. Aucun objectif ne semble prévaloir sur les autres, plusieurs accordent une importance moyenne à chaque objectif proposé. Ce type de dirigeants représente $25 \%$ de l'échantillon.

Type 3 : Les «conservateurs anciens » : il s'agit de dirigeants de 50 ans et plus, très expérimentés, autodidactes ou de niveau secondaire, de type autre que gestionnaire, seuls $26 \%$ d'entre eux ont participé à un ou des stages de 
gestion récemment ; ils recherchent systématiquement en priorité la stabilité, la pérennité et l'indépendance. Ce type de dirigeants représente $37 \%$ de l'échantillon.

Au total, trois types de dirigeants, avec des caractéristiques d'âge, d'expérience, de formation et d'objectifs différentes, ont été identifiés. Il est clair que ces trois profils, qui globalement correspondent à ceux identifiés dans la littérature, donnent une vision très synthétique et assez réductrice de la réalité. Cette classification permet toutefois de mieux cerner la variété des dirigeants de PME de notre échantillon.

Analysons maintenant l'attitude de ces dirigeants face aux données comptables de gestion.

\subsection{Le système d'information d'une importante majorité de dirigeants de PME est en partie comptable}

Les résultats révèlent que, dans l'ensemble, les pratiques comptables en PME répondent à une norme minimale de complexité. Peu de dirigeants se dispensent de toute production et de toute utilisation des données comptables à des fins de gestion.

La note moyenne de complexité des SDCG observés (figure 5) est de 47,30 sur 80 , la note la plus basse étant de 14 , la plus élevée, de 79, l'écart type, de 15,21 et la médiane, de 48,5 . Seuls $2 \%$ des dirigeants de l'échantillon ne disposent d'aucune donnée d'analyse financière (analyse de la rentabilité et du risque de défaillance), seuls $8 \%$ n'élaborent jamais de situations intermédiaires et trois dirigeants sur quatre disposent d'un tableau de bord, d'un système de calculs de coûts ou d'un système de prévisions. En définitive, l'analyse de la

FIGURE 5

Degré de complexité des SDCG des 113 PME de l'échantilon

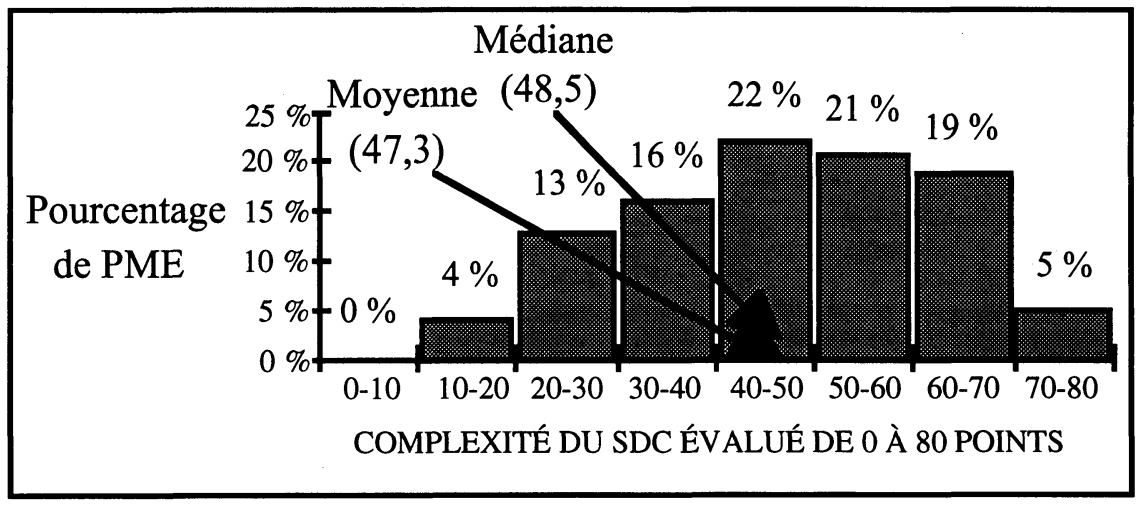


complexité des SDCG révèle que la plupart des dirigeants de PME ont accès à une base minimale de données comptables élaborées à des fins de gestion, mais qu'au-delà de celle-ci, la complexité des SDCG observés est assez hétérogène.

La note moyenne d'utilisation des données comptables à des fins de gestion par les dirigeants de PME (figure 6) est, quant à elle, de 44,63, la note la plus basse étant de 4,93, la plus élevée, de 76,64, l'écart type, de 14,28 et la médiane, de 44,93. Les résultats statistiques relatifs aux questions d'utilisation montrent que la quasi-totalité des dirigeants de PME travaillent avec les données comptables, seuls $4 \%$ d'entre eux déclarent ne jamais les utiliser ou presque (note inférieure à 20 ). De même, une faible fraction (12\%) déclare les utiliser de manière très importante (note supérieure à 60). En définitive, une grande majorité de dirigeants de PME utilise les données comptables plutôt faiblement ( $31 \%$ ont une note comprise entre 20 et 40 ) ou moyennement (53\% ont une note comprise entre 40 et 60 ). De ce fait, les affirmations selon lesquelles les chefs de PME disposent le plus souvent de SDCG rudimentaires systématiquement sous-utilisés ne correspondent pas à la réalité d'une grande majorité de PME.

\section{FIGURE 6}

\section{Degré d'utilisation des SDCG des données comptables par les 113 PME de l'échantilon}

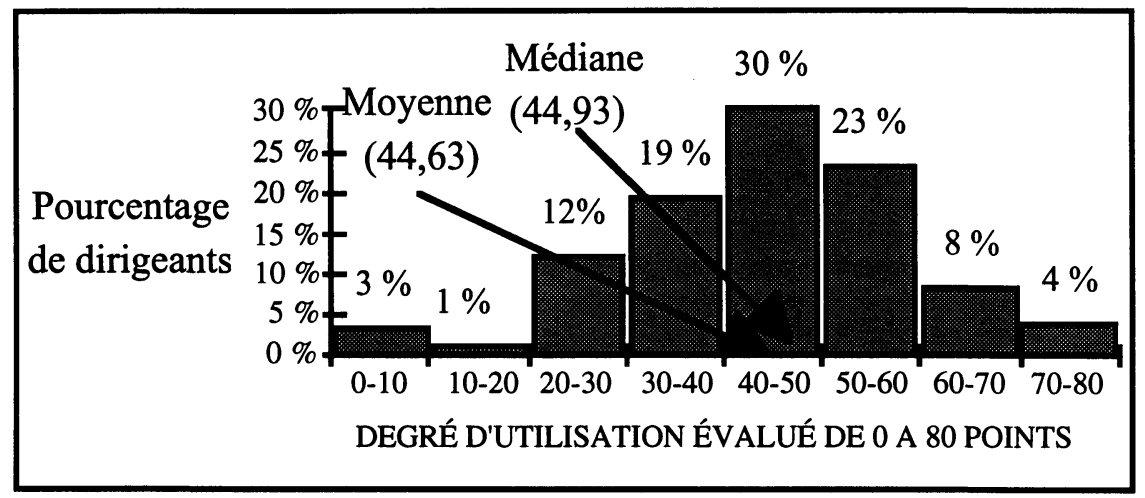

Au-delà de ce constat, l'analyse des relations entre données comptables disponibles et données comptables utilisées nous amène à conclure à la convergence des pratiques : ceux qui disposent des SDCG les plus complexes sont logiquement ceux qui utilisent le plus les données comptables (la relation entre les indicateurs globaux de complexité et d'utilisation, appréciée à travers le calcul d'un coefficient de corrélation, est significative à 99,9\%). La classification (réalisée sur la base de données fournies par une analyse en composantes principales dont les valeurs propres des deux premiers axes expliquent à elles 
seules $69,76 \%$ de l'inertie totale du nuage) confirme ce résultat, puisque le strict respect des règles de coupure de l'arbre au seuil des deux nœuds consécutifs entre lesquels la variation de l'indice de niveau est la plus forte ne fait apparaître que deux principaux types de pratiques comptables (définies simultanément en termes de production et d'utilisation). Ce sont les suivants :

Type 1 : «Les pratiques comptables de gestion fortes ou très fortes »: il s'agit de dirigeants disposant de SDCG de complexité élevée ou très élevée et d'un degré d'utilisation plutôt important ou très important. Ils représentent $54 \%$ de l'échantillon.

Type 2 : «Les pratiques comptables de gestion faibles ou très faibles » : il s'agit de dirigeants disposant de SDCG de complexité faible et d'un degré d'utilisation plutôt faible, parfois même très faible. Ils représentent $46 \%$ de l'échantillon.

Les scores moyens obtenus par les dirigeants de chacun des deux types, sur chacune des variables et sous-variables caractérisant les pratiques comptables de gestion, confirment cette interprétation. Les scores obtenus par les dirigeants de la classe 1 (pratiques comptables fortes ou très fortes) sont en effet nettement supérieurs à ceux obtenus par les dirigeants de la classe 2 (pratiques comptables faibles ou très faibles), à la fois pour les variables relatives à la complexité du SDCG et pour celles relatives à l'utilisation des données comptables (tableau 2).

TABLEAU 2

Scores moyens obtenus par les dirigeants de chacun des deux types de pratiques comptables de gestion

\begin{tabular}{lccc}
\hline & $\begin{array}{c}\text { Moyenne des dirigeants } \\
\text { ayant des pratiques } \\
\text { comptables de type 1 }\end{array}$ & $\begin{array}{c}\text { Moyenne des dirigeants } \\
\text { ayant des pratiques } \\
\text { comptables de type 2 }\end{array}$ & $\begin{array}{c}\text { Moyenne générale } \\
\text { des 113 dirigeants }\end{array}$ \\
\hline Délai de production /20 & 14,92 & 9,28 & 12,32 \\
Fréquence de production /20 & 14,83 & 7,89 & 11,64 \\
Diversité de production /20 & 14,01 & 8,92 & 11,67 \\
Détail de production /20 & 14,79 & 8,02 & 11,67 \\
Degré global de complexité /80 & 58,54 & 34,12 & 47,30 \\
Fréquence d'utilisation /20 & 14,31 & 8,85 & 11,80 \\
Durée d'utilisation /20 & 13,93 & 8,37 & 11,37 \\
Intensité d'utilisation /20 & 13,06 & 9,48 & 11,42 \\
Diversité d'utilisation /20 & 11,73 & 8,08 & 10,05 \\
Degré global d'utilisation /80 & 53,04 & 34,77 & 44,63 \\
\hline
\end{tabular}


Au total, les systèmes de données comptables mis à la disposition des dirigeants de PME semblent pouvoir être qualifiés de systèmes d'information comptable, autrement dit, quand les données existent, elles sont le plus souvent utilisées et peuvent donc être considérées comme des informations du point de vue du dirigeant.

Il convient maintenant de procéder à une analyse des relations entre les profils des dirigeants repérés et leurs pratiques comptables de gestion afin de déterminer si à chaque profil identifié correspondent (ou non) des pratiques spécifiques.

\subsection{La mise en évidence de liens significatifs entre le profil des dirigeants et leurs pratiques comptables de gestion}

Les résultats font apparaître une relation significative entre les profils de dirigeants identifiés et les types de pratiques comptables. Une analyse plus approfondie démontre que ces relations existent à la fois avec la complexité des SDCG et avec le degré d'utilisation des données comptables de gestion par les dirigeants.

Une relation significative apparaît entre le profil des dirigeants et les types de pratiques comptables de gestion.

TABLEAU 3

Degré de signification du test du khi ${ }^{2}$

entre le profil des dirigeants et les types de pratiques comptables

Types de pratiques comptables

Profil des dirigeants

$99,5 \%$

Le tableau ci-dessous présente, pour chaque profil, les pourcentages de dirigeants appartenant à chacun des deux types de pratiques comptables de gestion.

TABLEAU 4

Répartition des dirigeants de chaque profil dans chacun des deux types de pratiques comptables

\begin{tabular}{lcc}
\hline & $\begin{array}{c}\text { Pratiques comptables } \\
\text { fortes ou très fortes }\end{array}$ & $\begin{array}{c}\text { Pratiques comptables } \\
\text { faibles ou très faibles }\end{array}$ \\
\hline Les « managers ambitieux» & $72,09 \%$ & $27,91 \%$ \\
Les « débutants incertains » & $57,14 \%$ & $42,86 \%$ \\
Les « conservateurs anciens » & $33,33 \%$ & $66,67 \%$ \\
\hline
\end{tabular}


Il apparaît que les deux tiers des « conservateurs anciens » (dirigeants de type 3) ont des pratiques comptables de gestion «faibles ou très faibles », c'est-à-dire qu'ils disposent de SDCG de complexité faible et affichent un degré d'utilisation plutôt faible, parfois très faible, et qu'en revanche, une légère majorité des « débutants incertains » (dirigeants de type 2) et une grande majorité des «managers ambitieux » (dirigeants de type 1) ont des pratiques comptables de gestion « fortes ou très fortes », c'est-à-dire qu'ils disposent de SDCG de complexité élevée ou très élevée et ont un degré d'utilisation plutôt important ou très important.

Des relations significatives existent de même entre le profil des dirigeants et la complexité des SDCG.

TABleau 5

Degré de signification des tests du $\mathbf{k h i}^{2}$ entre le profil des dirigeants et les variables et sous-variables caractérisant la complexité du SDCG

\begin{tabular}{lccccc}
\hline & Délai & Fréquence & Diversité & Détail & $\begin{array}{c}\text { Complexité } \\
\text { du SDCG }\end{array}$ \\
\hline Profil des dirigeants & NS & $99,5 \%$ & $95 \%$ & $99,5 \%$ & $99 \%$ \\
\hline
\end{tabular}

NS $=$ Non significatif

Il apparaît clairement que les « managers ambitieux » disposent de données comptables plus diverses, plus détaillées et élaborées plus fréquemment que les « conservateurs anciens ». En revanche, les « débutants incertains » disposent de SDCG de complexité hétérogène. L'analyse des relations entre le profil des dirigeants et les types de techniques comptables confirme, dans l'ensemble, l'existence d'une relation forte.

TABLEAU 6

Degré de signification des tests du khi ${ }^{2}$ entre le profil des dirigeants et les types de techniques comptables

\begin{tabular}{ccccccccc}
\hline & $\begin{array}{c}\text { Comptabilité } \\
\text { générale }\end{array}$ & $\begin{array}{c}\text { Contrôle } \\
\text { de gestion }\end{array}$ & $\begin{array}{c}\text { Comptabilité } \\
\text { analytique }\end{array}$ & $\begin{array}{c}\text { Gestion } \\
\text { budgétaire }\end{array}$ & $\begin{array}{c}\text { Analyse } \\
\text { financière }\end{array}$ & $\begin{array}{c}\text { Analyse de } \\
\text { rentabilité }\end{array}$ & $\begin{array}{c}\text { Analyse } \\
\text { du risque }\end{array}$ & $\begin{array}{c}\text { Tableau } \\
\text { de bord }\end{array}$ \\
\hline $\begin{array}{l}\text { Profil des } \\
\text { dirigeants }\end{array}$ & NS & $99 \%$ & NS & $99,5 \%$ & $99 \%$ & $99 \%$ & $\begin{array}{c}\text { NS } \\
(90 \%)\end{array}$ & $99,95 \%$ \\
\hline
\end{tabular}

Aucune relation significative n'apparaît entre le profil des dirigeants et les pratiques de comptabilité générale, de comptabilité analytique et d'analyse du risque. De telles relations existent en revanche entre le profil des dirigeants 
et les autres types de techniques comptables. Les « managers ambitieux » ont, pour la plupart, des pratiques plus complexes que les «conservateurs anciens » en matière de contrôle de gestion (et plus précisément de gestion budgétaire), d'analyse financière (particulièrement en analyse de rentabilité) et d'élaboration de tableaux de bord. Les « débutants incertains » ont des pratiques hétérogènes.

Une relation significative existe enfin entre le profil des dirigeants et le degré global d'utilisation des données comptables de gestion.

TABLEAU 7

Degré de signification des tests du $\mathbf{k h i}^{2}$

entre le profil des dirigeants et les variables et sous-variables caractérisant leur utilisation des données comptables

\begin{tabular}{lccccc}
\hline & $\begin{array}{c}\text { Fréquence } \\
\text { d'utilisation }\end{array}$ & $\begin{array}{c}\text { Durée } \\
\text { hebdomadaire }\end{array}$ & $\begin{array}{c}\text { Intensité } \\
\text { d'utilisation }\end{array}$ & $\begin{array}{c}\text { Diversité } \\
\text { d'utilisation }\end{array}$ & $\begin{array}{c}\text { Degré } \\
\text { d'utilisation }\end{array}$ \\
\hline Profil des dirigeants & NS $(90 \%)$ & NS & $99 \%$ & $99,5 \%$ & $99 \%$ \\
\hline
\end{tabular}

Aucune relation significative n'apparaît entre le profil des dirigeants, d'une part, et la fréquence ainsi que la durée moyenne d'utilisation des données comptables, $d$ 'autre part. Cependant, le profil des dirigeants est significativement associé aux sous-variables caractérisant les situations d'utilisation (intensité et diversité) et au degré global d'utilisation des données comptables de gestion. Les «managers ambitieux » utilisent les données comptables dans des situations plus diverses et, pour chacune de ces situations, plus intensément que «les conservateurs anciens ». En résumé, ils utilisent globalement plus les données comptables que ces derniers, et les «débutants incertains » les utilisent de manière hétérogène.

Au total, l'analyse des résultats laisse clairement apparaître qu'en règle générale, à chaque profil de dirigeants est associé un type de pratiques comptables de gestion spécifique : les SDCG rudimentaires et peu utilisés appartiennent le plus souvent à des dirigeants de type «conservateurs anciens », les dirigeants de type «managers ambitieux » semblent disposer de modèles de gestion plus structurés, et les «débutants incertains » ont des pratiques comptables hétérogènes.

\section{Conclusion}

Dans un premier temps, une classification hiérarchique, opérée sur les données relatives aux caractéristiques des dirigeants de PME, a permis d'identifier trois profils de dirigeants avec différentes caractéristiques d'âge, d'expérience, de 
formation et d'objectifs : les «managers ambitieux», les «conservateurs anciens » et les « débutants incertains ».

Ensuite, l'analyse descriptive des pratiques comptables des dirigeants de PME souligne que le système d'information d'une importante majorité de dirigeants observés est en partie comptable et que, lorsque les données existent, elles sont le plus souvent utilisées et peuvent donc être considérées comme des informations du point de vue du dirigeant. L'étude contribue donc sur ce point à dissiper l'ambiguïté présente dans la littérature. Par ailleurs, la classification hiérarchique réalisée sur les données relatives aux comportements comptables des dirigeants de PME ne fait apparaître que deux principaux types de pratiques comptables de gestion: des pratiques comptables faibles ou très faibles, et des pratiques comptables fortes ou très fortes.

Enfin, l'analyse des relations entre les profils relevés et les pratiques comptables a permis d'avancer que le profil des dirigeants est significativement lié à leur type de pratiques comptables. Les dirigeants de type «conservateurs anciens » ont le plus souvent des pratiques comptables faibles ou très faibles et une grande majorité des dirigeants de type «managers ambitieux » ont des pratiques comptables fortes ou très fortes. Les «débutants incertains » ont, quant à eux, des pratiques comptables hétérogènes.

$\mathrm{Au}$ total, ces résultats mettent une fois encore en évidence une distinction entre «logique patrimoniale » et «logique entrepreneuriale» (Julien et Marchesnay, 1988). Ils confirment en outre l'influence profonde des composantes du profil du dirigeant sur les modes de gestion de la PME et par là même, le rôle central du chef de PME sur la conduite de sa firme (d'Amboise, 1989 ; Marchesnay, 1992b ; Lacombe-Saboly, 1994).

La prudence doit, cependant, présider à l'interprétation et à l'appréciation de nos conclusions compte tenu des difficultés et des limites de l'étude. Ces limites sont de plusieurs ordres. Elles sont tout d'abord liées à la taille de l'échantillon : un échantillon de 113 observations peut être considéré comme assez important pour le chercheur en gestion, mais le statisticien le trouvera trop limité. En outre, notre travail relève l'existence de cas « atypiques », mais la méthodologie employée ne permet pas de développer d'explications approfondies sur ces situations «anormales»; il serait nécessaire pour cela de revenir sur le terrain. Enfin, le caractère synchronique et statique de l'étude ne permet pas d'analyser les phénomènes d'évolution.

De façon générale, ces limites indiquent clairement quelques directions de prolongements pour notre recherche et, à notre sens, un approfondissement de la connaissance du comportement comptable des dirigeants de PME passe tout d'abord par un élargissement du modèle de recherche pour affiner et 
compléter certaines de nos conclusions. Le dirigeant de PME pourra notamment être caractérisé par des variables plus complexes et plus subjectives que celles utilisées dans notre étude, les aspects psychologiques de sa personnalité (son attitude face au risque, à l'incertitude ; sa culture, sa créativité, etc.) pourront, en effet, être intégrés au modèle.

Elle passe ensuite par une multiplication d'analyses plus en profondeur dans les PME. Il est prévu de compléter l'enquête réalisée par une étude qualitative. Celle-ci devrait permettre une meilleure représentation, une meilleure perception des phénomènes étudiés en rendant possible une étude plus approfondie des jeux d'acteur. De même, une étude longitudinale, menée dans un nombre limité d'entreprises, permettrait de dépasser la saisie à un moment donné de l'état de la situation.

En définitive, si cette étude a pu confirmer, infirmer ou compléter les conclusions de recherches antérieures, elle ne constitue bien évidemment qu'un propos d'étapes. En effet, l'analyse des résultats souligne la nécessité d'approfondir et d'élargir sa problématique de base, et de multiplier les contextes d'études. Ces prolongements devraient permettre d'enrichir les représentations et les explications amorcées dans ce travail et ainsi de mieux comprendre la spécificité des schémas de gestion des dirigeants de PME.

\section{Bibliographie}

ABDou, H. (1991), «L'influence de l'évolution des systèmes de production sur le système d'information comptable: étude empirique», Thèse de doctorat de sciences de gestion, Université de Montpellier II.

BAteson, G. (1977), Vers une écologie de l'esprit, Paris, Éditions du Seuil.

BLAIS, R.A. et J.M. ToulousE (1990), «Les motivations des entrepreneurs : une étude empirique de 2278 fondateurs d'entreprises dans 14 pays ", Revue Internationale $P M E$, vol. 3, nos 3-4, p. 268-300.

Carland, J.W., J.A.C. Carland et C.D. Aby Jr. (1989), «An assessment of the psychological determinants of planning in small businesses ", International Small Business Journal, vol. 7, $\mathrm{n}^{\circ}$ 4, juillet-septembre, p. 23-34.

Chapellier, P. et C. DE Mongolfier (1995), « Une synthèse des approches méthodologiques du système d'information comptable», $16^{\mathrm{e}}$ Congrès de l'Association française de comptabilité, Tome 2, Montpellier, mai, p. 1053-1069.

Chapellier, P. (1994), «Comptabilités et système d'information du dirigeant de PME : essai d'observation et d'interprétation des pratiques », Thèse de doctorat de sciences de gestion, Université de Montpellier II.

Crozier, M. et E. Friedberg (1977), L'acteur et le système, Paris, Éditions du Seuil. 
D’ Amboise, G. et A. BAKANiBona (1990), «La planification dans les PME: une synthèse de résultats empiriques, conclusions et recommandations », Revue Internationale PME, p. 147-166.

D'AMBOISE, G. (1989), «Planification stratégique dans les PME : des modèles émergents de la littérature », Revue Internationale de Gestion des PMO, vol. 4, no 2, p. 46-57.

Davis, G.B., M.H. Olson, J.A. Ajenstat et J.L. Peaucelle (1986), Systèmes d'information pour le management, Montréal, Éditions G. Vermette Inc., Paris, Economica (Volume 1 : Les bases et Volume 2: Les approfondissements).

DE ThomAs, A.R. et W.B. FREDENBERGER, (1985), « Accounting needs of very small business », CPA Journal, no $55, \mathrm{p} .14-23$.

DUPUY, Y. (1987), «Vers de nouveaux systèmes d'information pour le chef de petite entreprise? (Quelques difficultés de dépassement de l'information comptable) », Papier de recherche CREGO, IAE, Université de Montpellier II.

DupuY, Y. (1994), «Une lecture des recherches actuelles en comptabilité-contrôle», Annales du Management, XII ${ }^{\mathrm{e}}$ Journées nationales des IAE, Montpellier, 2 et 3 février, p. 17-33.

ELMORE, R. (1986), «A contingency theory approach to an empirical classification of management accounting information systems », Thèse de doctorat, Université du Mississipi.

Evrard, Y., B. Pras, E. Roux, J.M. Choffray et A.M. Dussay (1993), Market : études et recherches en marketing, fondements, méthodes, Paris, Nathan.

FALLERY, B. (1983a), «Un système d'information pour les PME », Revue Française de Gestion, novembre-décembre, p. 70-76.

FALLERY, B. (1983b), «Le système d'information du dirigeant de petite entreprise », Thèse de doctorat de $3^{\mathrm{e}}$ cycle, spécialité : analyse industrielle, Université de Montpellier I.

Fenneteau, H. et C. Biales (1993), Analyse statistique des données, applications et cas pour le marketing, Paris, Ellipses.

FERnANDEZ, V., C PICORY et F. ROWE (1994), «Diversité, cohérence et pertinence des outils de gestion : le cas des PME d'Île-de-France », Actes de la $39^{e}$ Conférence mondiale des PME, Strasbourg, 27-29 juin.

GABLE, M. et M.T. TOPOL (1987), «Planning practices of small-scale retailers », American Journal of Small Business, vol. 12, n 2, p. 19-32.

GASSE, Y. (1989), «L'utilisation de diverses techniques et pratiques de gestion dans la $\mathrm{PME} »$, Revue Internationale de Gestion des $P M O$, vol. 4, n 1 , p. 3-11.

HAMILTON, R.T. (1987), « Motivations and aspirations of business founders », International Small Business Journal, vol. 6, $\mathrm{n}^{0}$ 1, automne, p. 69-78. 
HESS, D.W. (1987), «Relevance of small business courses to management needs », Journal of Small Business Management, janvier, p. 26-34.

HolmES, S. et D. NiCHOLLS (1988), «An analysis of the use of accounting information by Australian small business », Journal of Small Business Management, avril, p. 57-68.

Holmes, S. et D. Nicholls (1989), «Modelling the accounting information requirements of small businesses », Accounting and Business Research, vol. 19, $\mathrm{n}^{0}$ 74, printemps, p. 143-150.

Holmes, S., G. Kelly et R. Cunningham (1991), "The small firm information cycle : a reappraisal », International Small Business Journal, vol. 9, $\mathrm{n}^{\circ} 2$, janvier-mars, p. 40-53.

Julien, P.A. et M. MÁrhesnay (1988), La petite entreprise, Paris, Éditions Vuibert.

JULIEN, P.A. et M. MARCHESNAY (1990), «Small business as a transaction space», Entrepreneurship and Regional Development, vol. 2, $\mathrm{n}^{0} 3$.

KANDHWALLA, P.N. (1972), «The effects of different types of competition on the use of managerial controls ", Journal of Accounting Research, automne.

LACOMBE-SABOLY, M. (1994), «Les déterminants de la qualité des produits comptables des entreprises : le rôle du dirigeant », Thèse de doctorat, Université de Poitiers.

LAUFER, J. (1974), « Comment on devient entrepreneur », Revue Française de Gestion, $\mathrm{n}^{0} 2$.

LEFEBVRE, E. (1991), «Profil distinctif des dirigeants de PME innovatrices », Revue Internationale PME, vol. 4, $\mathrm{n}^{\circ} 3$, p. 7-26.

LORRAIN, J. et L. DUSSAULT (1988), «Les entrepreneurs artisans et opportunistes : une comparaison de leurs comportements de gestion », Revue Internationale $P M E$, vol. 1, $\mathrm{n}^{\circ} 2$, décembre, p. 157-176.

LUCAS, H.C. Jr. (1975), «Performance and the use of an information system », Management Science, vol. 21, no 8, avril, p. 908-919.

MACINTOSH, N.B. (1987), «Les différences individuelles et l'utilisation des systèmes comptables », Actes du $8^{e}$ Congrès de l'Association française de comptabilité, Rennes, avril, p. 24-27

MARCHESNAY, M. (1982), «Pour un modèle d'hypofirme », dans Entreprises et Organisations, Mélanges en l'honneur de madame le professeur Jane Aubert-Krier, Paris, Economica.

MARCHESNAY, M. (1988), «La mercatique de la petite entreprise », Revue Interna-

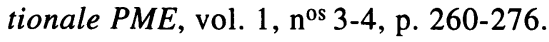

MARChESNAY, M. (1990), Économie d'entreprise, Paris, Éditions Eyrolles. 
MARCHESNAY, M. (1992a), «L'offre de services en gestion à la petite entreprise», dans Mélanges en l'honneur du professeur Merigot, Paris, Economica.

Marchesnay, M. (1992b), «La PME: une gestion spécifique?», Problèmes Économiques, $\mathrm{n}^{0} 2276$, mai.

MARChESNAY, M. (1993), Management Stratégique, Paris, Éditions Eyrolles, Collection Gestion.

Martel, J.M., R. NAdeau, D. Elsliger et A. Guay (1985), «Les processus de décision des gestionnaires dans les PME québécoises : une étude exploratoire », Cahiers du Groupe de recherche sur l'aide à la décision, GRAD RR-48, Université Laval.

MÉLÈSE, J. (1979), «Information, besoins et désirs », Informatique et Gestion, n 105 , avril, p. 26-31.

MiNTZBERG, H. (1976), «Planning on the left and managing on the right », Harvard Business Review, juillet-août, p. 49-58.

MintzBerG, H. (1986), Le pouvoir dans les organisations, Paris, Les Éditions d'Organisation.

MINTZBERG, H. (1988), «Formons des managers, non des MBA ! », Harvard L'Expansion, hiver 1988-1989, p. 84-92.

MinTZBERG, H. (1990), Le manager au quotidien : les dix rôles du cadre, Paris, Les Éditions d'Organisation ( $4^{\mathrm{e}}$ impression).

Nadeau, R., J.J. Martel, C. Martel et B. M'Zali (1988), «L'utilisation des méthodes quantitatives pour les décisions de gestion dans les PME québécoises : une étude empirique », Papier de recherche Université de Laval, GRADE RR 88, Québec, novembre.

NADEAU, R., J.M. MARTEL et D. Bouyssou (1987), «L'utilisation des méthodes quantitatives pour l'aide à la décision dans les PME : rêve ou réalité ? », Gestion, février, p. 23-30.

NARO, G. (1989), «Facteurs et comportements d'embauche dans la PME », Thèse de doctorat, Université de Montpellier I.

NELSON, G.W. (1987), «Information needs of female entrepreneurs », Journal of Small Business Management, juillet, p. 38-44.

ORPEN, C. (1985), «The effects of long-range planning on small business performance : a further examination », Journal of Small Business Management, janvier, p. 16-23.

RASOLOFOSON, M.T. (1993), «Le système de contrôle interne dans la petite entreprise, la perception des dirigeants », Actes du congrès international francophone de la PME (CIFPME), Carthage, octobre. 
RAYMOND, L. (1988a), «L'impact des tâches critiques du gestionnaire de PME sur le succès d'un système d'information organisationnel », Revue Internationale $P M E$, vol. $1, \mathrm{n}^{\circ} 1$, septembre, p. 77-95.

RAYMOND, L. (1988b), «The impact of computer training on the attitudes and usage behavior of small business managers », Journal of Small Business Management, juillet, p. 8-13.

REIX, R. (1981), «Processus de décision et utilisation de l'information », Mélanges offerts à Pierre Vigreux, I.P.A. Toulouse, p. 677-697.

REIX, R. (1984), «Quelques facteurs affectant l'utilisation d'informations de caractère comptable », Actes du congrès de l'Association française de comptabilité, «Comptabilité et décision», Nice, avril, p. 357-370.

REIX, R. (1987), «Les problématiques d'évaluation des systèmes d'information », Actes du $8^{e}$ Congrès de l'Association française de comptabilité, Rennes, avril.

RIGGS, W.E. et J.S. BRACKER (1986), «Operations management and financial performance », American Journal of Small Business, vol. 10, $\mathrm{n}^{\mathrm{0}} 3, \mathrm{p} .17-23$.

Robinson, R.B. et J.A. PEARCE (1984), «Research thrusts in small firm strategic planning », Academy of Management Review, vol. 9, n 1, p. 128-137.

RobINSON, R.B., J.E. LOGAN et M.Y. SALEM (1986a), « Strategic versus operational planning in small retail firms », American Journal of Small Business, vol. 10, $\mathrm{n}^{\circ} 3$, p. 7-16.

Robinson, R.B., M.Y. Salem, J.E. Logan et J.N. PearCe (1986b), «Planning activities related to independent retail firm performance », American Journal of Small Business, vol. 11, $\mathrm{n}^{0} 1$, p. 19-26.

RoSENZWEIG, K.Y. (1977), "Effects of company organization structure on the accounting system », Thèse de doctorat, Université du Michigan.

SIMON, H.A. (1974), La science des systèmes, science de l'artificiel, Paris, Éditions EPI.

SIMON, H.A. (1987), "Making management decisions : the role of intuition and emotion », Academy of Management Executive, vol. 1, n 1, février, p. 57-64.

SLATTER, S., R. RANSLEY et E. WoodS (1988), «USM chief executives : do they fit the entrepreneurial stereotype?», International Small Business Journal, vol. $6, n^{0} 3$, avril-juin.

SMITH, N.R. (1967), «The entrepreneur and his firm: the relationship between type of man and type of company », East Lansing, Michigan State University, Graduate School of Business Administration.

SMITH, N.R., J.B. MINER et J.S. BRACKER (1992), « Defining the inventor-entrepreneur in the context of established typologies », Journal of Business Venturing, vol. 7, $\mathrm{n}^{\circ}$ 2, mars, p. 103-113. 
Solomon, G.T. et L.W. Fernald (1988), «Value profiles of male and female entrepreneurs », International Small Business Journal, vol. 6, $\mathrm{n}^{\circ} 3$, avril-juin, p. 23-33.

SPECHT, P.H. (1987), «Information sources used for strategic planning decisions in small firms », American Journal of Small Business, vol. 11, $\mathrm{n}^{0} 4$, printemps, p. 27-34.

TAGIURI, R. et J.A.. DAVIS (1992), «On the goals of successful family companies », Family Business Review, vol. 5, $\mathrm{n}^{0} 1$, printemps, p. 43-62.

Woo, C.Y., A.C. Cooper et W.C. Dunkelberg (1991), «The development and interpretation of entrepreneurial typologies », Journal of Business Venturing, vol. $6, \mathrm{n}^{\circ} 2$, mars, p. 93-111. 


\section{ANNEXES}

Annexe A :

Extrait du questionnaire : « Caractérisation de l'état du système de données comptables » (questions 15 à 44)

A) Pratiques de comptabilité générale

15 / Combien de mois après la date de clôture disposez-vous d'un bilan et d'un compte de résultat utilisables à des fins de gestion?

- Moins d'un mois

- De 1 à 2 mois

- De 2 à 3 mois

- De 3 à 4 mois

- Plus de 4 mois

16 / L'établissement d'une situation intermédiaire se fait :

- Tous les mois

- Tous les trimestres

- Tous les semestres

- Ponctuellement (précisez) $\square$

- Jamais

17 / L'élaboration des situations intermédiaires présente un caractère plutôt :

- Complet et détaillé

- Général et approximatif

18 / Quels sont les délais nécessaires à l'établissement des situations intermédiaires?

- Moins d'un mois

- De 1 à 2 mois

- Plus de 2 mois

B) Pratiques de comptabilité analytique

19 / Existe-t-il, dans votre entreprise, un système de calcul de coûts ?

Oui Non

Si la réponse est Non, veuillez passer directement à la question 24 . 
20 / Votre entreprise dispose-t-elle des informations suivantes?

\begin{tabular}{lll}
\hline & OUI & NON \\
\hline Coûts fonctionnels globaux & & \\
\hline Coûts détaillés par produit ou par chantier & \\
\hline Coûts détaillés par atelier ou par service & \\
\hline
\end{tabular}

21 / Quels types de coûts calculez-vous?

\begin{tabular}{lll}
\hline Coût complet & OUI & NON \\
\hline Coût direct & \\
\hline Coût variable & \\
\hline Coût marginal & \\
\hline Autres (précisez) & \\
\hline
\end{tabular}

22 / Calculez-vous votre seuil de rentabilité ?

$\begin{array}{cc}\text { Oui } & \text { Non } \\ \square & \square\end{array}$

23 / Quelle est, dans le domaine des coûts, la fréquence de traitement des données réelles?

- Annuelle

- Semestrielle

$\square$

- Trimestrielle

$\square$

- Mensuelle

- Ponctuelle (précisez)

C) Pratiques budgétaires et de gestion prévisionnelle

24 / Existe-t-il dans votre entreprise un système de budget ou de prévisions?

Oui Non

Si la réponse est Non, veuillez passer directement à la question 32. 
25 / Les activités ou fonctions suivantes font-elles l'objet de budgets ou de prévisions?

OUI

NON

Achat, approvisionnement

Production, fabrication

Vente, distribution

Investissement, projet

Autres (précisez)

26 / Élaborez-vous un bilan et/ou un compte de résultat prévisionnel(s)?

Oui Non

27 / Votre cadre budgétaire est :

- Global

- Ventilé par activité

- Ventilé par atelier ou par service

28 / Vos prévisions couvrent une période :

- Annuelle

- Semestrielle

- Trimestrielle

- Mensuelle

- Autres (précisez)

29 / Les révisions de budgets ou de prévisions en cours d'année sont :

- Régulières

- Exceptionnelles

- Exclues

30 / Les comparaisons entre les réalisations et les prévisions sont effectuées :

- Annuellement

- Semestriellement

- Trimestriellement

- Mensuellement

- Ponctuellement (précisez)

- Jamais 
31 / Compte tenu des causes d'écarts retenant l'attention, l'analyse des écarts entre le réel et le prévu vous semble :

- Complète

- Sélective

- Absente

D) Étude de la rentabilité

\section{2 / Disposez-vous :}

\section{OUI}

NON

Du tableau des soldes intermédiaires de gestion

Du calcul de la capacité d'autofinancement

\section{3 / Disposez-vous de ratios mesurant :}

\section{OUI}

NON

Le poids des achats par rapport au chiffre d'affaires

La rentabilité de l'activité

La rentabilité des capitaux investis

La rentabilité du personnel

La rentabilité du matériel

Le poids des charges financières

Autres (précisez)

34 / Procédez-vous à une analyse comparative de la rentabilité :

\begin{tabular}{lll}
\hline & OUI & NON \\
\hline Dans le temps (comparaison d'une année sur l'autre) & & \\
\hline Dans l'espace (comparaison avec d'autres entreprises) & & \\
\hline
\end{tabular}

\section{5 / Procédez-vous à :}

\section{OUI}

NON

Une analyse globale de la rentabilité

Une analyse par activité de la rentabilité 
36 / Quelle est, dans le domaine de l'information sur la rentabilité, la fréquence d'élaboration des données?

- Annuelle

- Semestrielle

- Trimestrielle

- Mensuelle

- Ponctuelle (précisez)

- Inexistante

E) Étude du risque de défaillance

37 / Disposez-vous :

\begin{tabular}{lll}
\hline & OUI & NON \\
\hline Du bilan fonctionnel & & \\
\hline Du tableau de financement & & \\
\hline Du besoin en fonds de roulement normatif (prévisionnel) & \\
\hline
\end{tabular}

38 / Disposez-vous de ratios mesurant :

OUI

NON

La solvabilité

L'autonomie financière

L'évolution du besoin en fonds de roulement

Le délai de rotation des stocks

Le délai de rotation des créances clients

Le délai de rotation des dettes fournisseurs

Autres (précisez)

39 / Quelle est, dans le domaine de l'information sur le risque, la fréquence d'élaboration des données?

- Annuelle

- Semestrielle

- Trimestrielle

- Mensuelle

- Ponctuelle (précisez)

- Inexistante 
F) Tenue d'un tableau de bord

40 / Tenez-vous un tableau de bord?

Oui Non

Si la réponse est Non, passez directement à la question 43 .

41 / Celui-ci est mis à jour :

- Toutes les semaines

- Tous les mois

- Tous les trimestres

- Tous les semestres

- Tous les ans

- Autres (précisez)

42 / Quelles sont les principales informations contenues dans celui-ci ?

G) Pratiques de choix d'investissement et de financement

43 / Quand vous procédez à un investissement, vous avez à votre disposition :

\begin{tabular}{lccc}
\hline Fréquence d'élaboration & $\begin{array}{c}\text { À chaque } \\
\text { investissement }\end{array}$ & $\begin{array}{c}\text { Parfois mais } \\
\text { pas toujours }\end{array}$ & Jamais \\
\hline
\end{tabular}

Outils de gestion

Le taux de rendement comptable

Le délai de récupération

La valeur actuelle nette (VAN)

Le taux interne de rentabilité (TIR)

44 / Quand vous avez à choisir un mode de financement (emprunts, crédit bail, fonds propres...) vous avez à votre disposition :

\begin{tabular}{llcr}
\hline Fréquence d'élaboration & $\begin{array}{l}\text { À chaque choix } \\
\text { de financement }\end{array}$ & $\begin{array}{c}\text { Parfois } \\
\text { mais pas toujours }\end{array}$ & Jamais \\
\hline
\end{tabular}

Outils de gestion

La valeur actuelle nette (VAN)

Le taux interne de rentabilité (TIR) 


\section{Annexe B}

Extrait du questionnaire :

«Mesure du degré d'utilisation des données comptables »

51 / Mesure de la fréquence et de la durée hebdomadaire moyenne de l'utilisation

\begin{tabular}{|c|c|c|c|c|c|c|c|}
\hline \multirow[b]{2}{*}{$\begin{array}{l}\text { Vous utilisez les informations } \\
\text { comptables suivantes : }\end{array}$} & \multirow[b]{2}{*}{$\begin{array}{l}\text { Toutes les } \\
\text { semaines }\end{array}$} & \multicolumn{3}{|c|}{ RÉGULIÈREMENT } & \multicolumn{3}{|c|}{ PONCTUELLEMENT } \\
\hline & & $\begin{array}{c}\text { Tous les } \\
\text { mois }\end{array}$ & $\begin{array}{l}\text { Tous les } \\
\text { trimestres }\end{array}$ & $\begin{array}{c}\text { Tous les } \\
\text { semestres }\end{array}$ & $\begin{array}{l}\text { Une fois } \\
\text { par an }\end{array}$ & $\begin{array}{l}\text { Uniquement } \\
\text { quand un } \\
\text { problème } \\
\text { précis } \\
\text { se pose }\end{array}$ & Jamais \\
\hline
\end{tabular}

Le bilan, le compte de résultat

et les situations intermédiaires

Les informations sur les coûts

Les informations sur les budgets

Les informations sur les écarts

Les informations sur la rentabilité

Les informations sur le risque

\begin{tabular}{|c|c|c|}
\hline $\begin{array}{l}\text { Vous utilisez les informations sur l'aide } \\
\text { au choix de financement et d'investissement }\end{array}$ & $\begin{array}{l}\text { À chaque investissement ou } \\
\text { choix de financement }\end{array}$ & $\begin{array}{l}\text { Parfois mais pas Jamais } \\
\text { à chaque fois }\end{array}$ \\
\hline
\end{tabular}
au choix de financement et d'investissement

\begin{tabular}{llcccc}
\hline $\begin{array}{l}\text { Combien d'heures par semaine } \\
\text { passez-vous à utiliser } \\
\text { l'information comptable? }\end{array}$ & Aucune & $\begin{array}{c}\text { Moins } \\
\text { d'une heure }\end{array}$ & $\begin{array}{c}\text { Entre } 1 \\
\text { et } 3 \text { heures }\end{array}$ & $\begin{array}{c}\text { Entre } \\
\mathbf{3} \text { et } 5 \text { heures }\end{array}$ & $\begin{array}{c}\text { Plus de } \\
5 \text { heures }\end{array}$ \\
\hline
\end{tabular}


52 / Mesure de la diversité et de l'intensité de l'utilisation : Dans quelle mesure utilisez-vous l'information comptable pour :

(Pondérez de 1 à 5 chacune des propositions suivantes en donnant le plus de points aux objectifs qui entrainent la plus grande utilisation : entourez 1 pour une utilisation nulle et 5 pour une utilisation très importante.)

- Prévoir la situation des semaines et des mois à venir (prévision du chiffre d'affaires, du résultat, de la trésorerie...).

$\begin{array}{lllll}1 & 2 & 3 & 4 & 5\end{array}$

- Prendre des décisions du type fixation des prix de vente, des marges, élaboration de devis...

Fixer des objectifs par référence aux années passées, suivre la réalisation de ces objectifs et prendre à temps, si nécessaire, des mesures correctives.

- Connaître l'évolution de la trésorerie pour éviter de trop gros découverts, le paiement d'agios trop élevés...

- Connaître le dû fournisseurs (pour tenter de négocier des remises, éviter de payer plusieurs fois...) et le dû clients (pour relancer les clients si nécessaire...).

- Choisir de maintenir, de renouveler, de moderniser le potentiel de production.

- Choisir de maintenir une activité, de l'abandonner, de la sous-traiter .

- Choisir d'investir dans une activité nouvelle.

- Choisir un mode de financement (emprunt, fonds propres, crédit bail, location...).

- Gérer les emprunts en cours (remboursement d'un emprunt par anticipation, rallongement du délai de remboursement d'un emprunt, transformation d'un découvert en emprunt...).

- Convaincre le banquier en cas de demande de crédit ou de découvert.

- Gérer les placements financiers (SICAV, actions, obligations...).

- Connaître l'évolution des performances de l'entreprise (rentabilité, risques, coûts...) pour détecter toute éventuelle dégradation et intervenir rapidement en cas de nécessité.

- Surveiller l'évolution des performances de l'entreprise par rapport à celle des concurrents, par rapport aux normes de la profession. 


\section{Annexe C}

Caractérisation des variables du dirigeant

\begin{tabular}{ll}
\hline Variables & \multicolumn{1}{c}{ Eléments de caractérisation } \\
\hline Âge du dirigeant & - Moins de 30 ans; de 30 à 39 ans ; \\
& de 40 à 49 ans ; de 50 à 59 ans ; \\
& de 60 à 69 ans ; 70 ans et plus \\
\hline Expérience du dirigeant & - Temps (exprimé en années) passé \\
& par le dirigeant à la tête d'une entreprise \\
\hline Niveau de formation & Autodidacte ; CAP, BEP, BP; Baccalauréat; \\
& Bac + 2 (DEUG, BTS, DUT); Licence; \\
& Maîtrise ; Grande école ; Expertise comptable; \\
\hline Type de formation & $3^{e}$ cycle \\
\hline Suivi de stages de gestion & Gestionnaire/non gestionnaire \\
\hline
\end{tabular}

Mesure des buts poursuivis par le dirigeant : Quels sont les buts que vous poursuivez en priorité dans votre métier de chef d'entreprise? Veuillez répartir 100 points entre les quatre propositions de chacune des deux séries suivantes:

- Être le leader d'une entreprise de plus en plus importante.

- Ne pas perdre mon indépendance face aux organismes extérieurs (État, autres entreprises, banques...).

- Assurer la survie de mon entreprise.

- Gagner suffisamment d'argent pour m'assurer un niveau de vie satisfaisant.

- Éviter à l'entreprise des changements trop importants, maintenir la stabilité de la firme.

- Obtenir une croissance maximale du niveau d'activité, gagner des parts de marché.

- Être mon propre patron, jouir d'une pleine liberté.

- Réaliser un profit maximal. 